\title{
Magnetic focusing in triangular electron billiards
}

\author{
Bøggild, Peter; Kristensen, A.; Lindelof, Poul Erik
}

Published in:

Physical Review B Condensed Matter

Link to article, DOI:

10.1103/PhysRevB.59.13067

Publication date:

1999

Document Version

Publisher's PDF, also known as Version of record

Link back to DTU Orbit

Citation (APA):

Bøggild, P., Kristensen, A., \& Lindelof, P. E. (1999). Magnetic focusing in triangular electron billiards. Physical Review B Condensed Matter, 59(20), 13067-13072. https://doi.org/10.1103/PhysRevB.59.13067

\section{General rights}

Copyright and moral rights for the publications made accessible in the public portal are retained by the authors and/or other copyright owners and it is a condition of accessing publications that users recognise and abide by the legal requirements associated with these rights.

- Users may download and print one copy of any publication from the public portal for the purpose of private study or research.

- You may not further distribute the material or use it for any profit-making activity or commercial gain

- You may freely distribute the URL identifying the publication in the public portal

If you believe that this document breaches copyright please contact us providing details, and we will remove access to the work immediately and investigate your claim 


\title{
Magnetic focusing in triangular electron billiards
}

\author{
P. Bøggild \\ Mikroelektronik Centret, Technical University of Denmark, Building 345e, DK-2800 Lyngby, Denmark \\ A. Kristensen and P. E. Lindelof \\ Niels Bohr Institute, University of Copenhagen, Universitetsparken 5, DK-2100 Copenhagen Ф, Denmark
}

(Received 11 November 1998)

\begin{abstract}
The classical ballistic magnetotransport in triangular electron billiards fabricated in a high mobility GaAs heterostructure has been studied at $4.2 \mathrm{~K}$. The sample geometry may be viewed as a double-slit structure with a skewed injection angle. We observe a striking cancellation of the magnetic focusing spectrum compared to the case of a perpendicular injection angle. From numerical and analytical analysis, we confirm that the quenching is a fundamental geometrical effect, and identify two mechanisms responsible for the anomaly. The focusing spectrum of the considered skewed geometry is remarkably sensitive to the angular distribution of injected electrons as well as the overall injection angle. [S0163-1829(99)06619-9]
\end{abstract}

\section{INTRODUCTION}

The availability of high mobility electron systems and high resolution lithography techniques makes it possible to fabricate structures far smaller than the elastic mean free path and with tolerances comparable to the Fermi wavelength. Such structures are ideal for studies of ballistic transport effects. In the classical regime, where the elastic mean free path but not the coherence length exceeds the linear dimensions of the structure, various types of transport anomalies have been reported over the past decade. Interesting examples are quenching of the Hall effect, ${ }^{1}$ collimation $^{2,3}$ and non-Ohmic addition ${ }^{4}$ of quantum point contacts, electrostatic focusing, ${ }^{5}$ and magnetic focusing in double-slit geometries. ${ }^{6,7}$ Recently, renewed interest in these classical transport effects has emerged from their importance in the studies of composite fermions, ${ }^{8}$ and from their presence as background in interpretation of quantum interference experiments. ${ }^{9,10}$ Since quantum interference effects always appear at lower temperatures than the corresponding classical effects, it is often necessary to elevate the temperature to a few Kelvin in order to smear out the quantum effects relative to the classical effects.

The magnetic focusing of two-dimensional (2D) electrons in a double-slit geometry is a particularly robust example of classical ballistic transport. A current is passed through two openings in a wall, i.e., from an emitter to a collector. In a magnetic field perpendicular to the electron gas, the emitted electrons may perform skipping orbits on the walls, leading to resonant transmission whenever an integer number $p$ of cyclotron orbit diameters $2 R_{c}$ equals the distance $L$ between the openings [see Fig. 1(a)]. This gives rise to a periodic sequence of peaks in the magnetoconductance with the period

$$
\Delta B=\frac{2 \hbar k_{\mathrm{F}}}{e L},
$$

where $k_{F}$ is the Fermi wave number in the 2D electron gas (2DEG) and $e$ is the electronic charge.

Previous studies considered geometries with perpendicular injection angle, i.e., where the injection cone is centered around a direction normal to the wall. We report, experimentally as well as theoretically, the magnetic focusing effects in a skewed injection angle geometry, where the injection cone is tilted by an angle $\alpha_{0}=\pi / 3$ with respect to the normal, and find a number of new anomalies compared to the perpendicular injection angle geometry. We find the amplitude, shape, and period of the spectrum for a skewed geometry to depend not only on the injection angle itself, but also strongly on the shape of the angular distribution of emitted electrons. This anomalous focusing in skewed geometries arises from the competition of two ballistic mechanisms, focusing and aiming, which in geometries with perpendicular injection angle are practically indistinguishable. The distinction between focusing and aiming is geometrical rather than physical; it is the selection of injection angles that governs which of the mechanisms dominate the transport behavior.

\section{MEASUREMENTS}

The starting point for the experiments is a $\mathrm{GaAs} / \mathrm{GaAl}_{0.3} \mathrm{As}_{0.7}$ heterostructure with an electron mobility of $180 \mathrm{~m}^{2} / \mathrm{V} \mathrm{s}$ and a carrier density of $n=2.1 \times 10^{15} \mathrm{~m}^{-2}$ measured at $0.3 \mathrm{~K}$ in the dark. Three hexagonal aluminum electrodes are deposited on top of the heterostructure using electron-beam lithography. By applying a negative formation voltage $V_{f}=-0.3 \mathrm{~V}$ to the electrodes, a triangular electron billiard is formed in the electron gas. The billiard has open corners of lithographic widths $w_{0}$, while the actual width of the electron channel in the opening will be reduced to $w$ $<w_{0}$ at more negative gate voltages. Two such types of billiards have been used in this work: a small billiard A with the emitter-collector distance $L=0.7 \mu \mathrm{m}$ and a large billiard B with $L=1 \mu \mathrm{m}$ [see Fig. 1(e)]. The corresponding pinchoff voltages are $V_{A}=-0.50 \mathrm{~V}$ and $V_{B}=-1.0 \mathrm{~V}$. In an earlier study ${ }^{9}$ we have estimated the carrier densities near pinch 

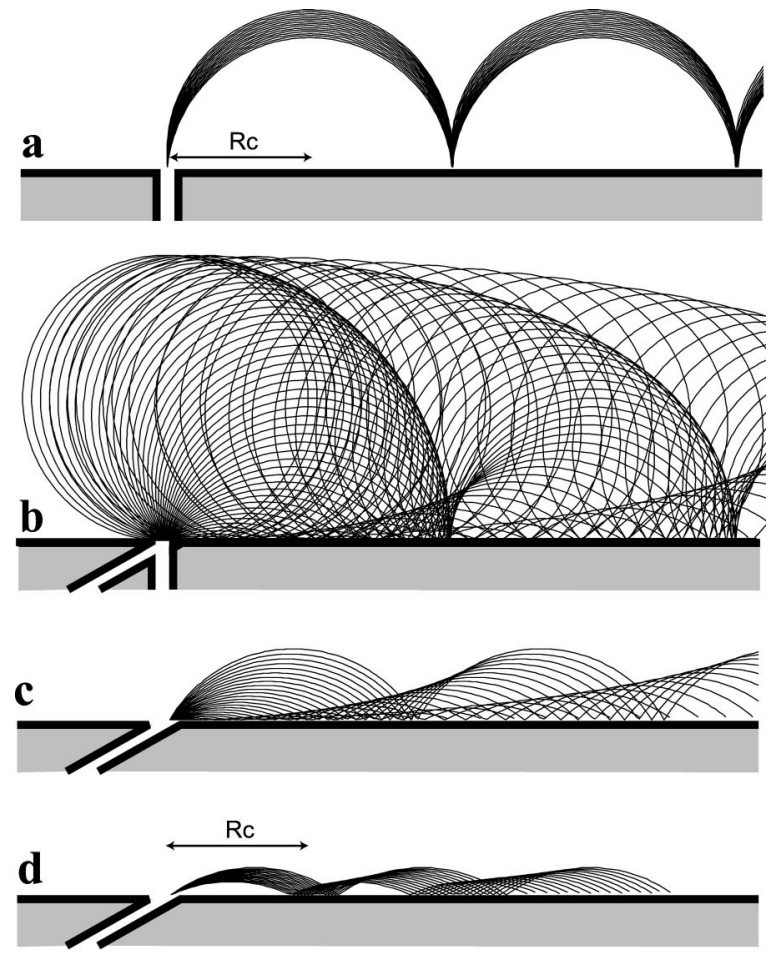

e
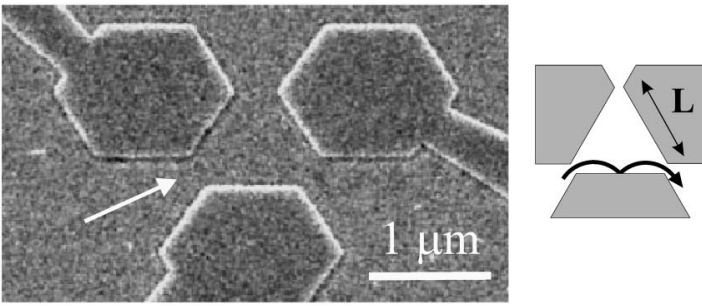

FIG. 1. A narrow distribution of electrons emitted through a hole in a specularly reflecting surface. (a) Pure aiming: in a perpendicular geometry $\left(\alpha_{0}=0, \alpha_{\max } \rightarrow 0\right)$ a narrow electron beam is reflected at points along the surface located at integer times $2 R_{c}$. (b) Pure focusing: in skewed and perpendicular geometries $\left(\alpha_{0}=0\right.$, $\alpha_{\max }=\pi / 2$ ): caustics are formed at the same reflection points as for the aiming. (c) Combined focusing and aiming effects in skewed geometry $\left(\alpha_{0}=\pi / 3, \alpha_{\max }=\pi / 6\right)$ : no clear focal points are seen. (d) Pure aiming effect in skewed geometry $\left(\alpha_{0}=\pi / 3, \alpha_{\max } \rightarrow 0\right)$ : focal points appear with half periods compared to pure focusing. The distance between the reflection points is halved. (e) Micrograph of sample, with the skewed injection indicated by white arrow. The thick white bar is $1 \mu \mathrm{m}$. The drawing in the bottom right illustrates skipping orbits in the triangular geometry.

off to $n_{A} \approx 0.7 \times 10^{15} \mathrm{~m}^{-2}$ and $n_{B} \approx 1.0 \times 10^{15} \mathrm{~m}^{-2}$. The carrier densities without applied gate voltage were, after processing, in the range $n_{A} \approx 1.3 \times 10^{15}$ and $n_{B} \approx 1.7$ $\times 10^{15} \mathrm{~m}^{-2}$, as obtained from the slope $1 /$ ne of the Hall resistance.

The samples were only cooled to $4.2 \mathrm{~K}$ where classical transport effects still dominate over quantum transport effects. ${ }^{9}$ The two-terminal ac differential resistance $(d V / d I)$ was measured with lock-in technique at fixed currents of 1 $\mathrm{nA}$ as a function of magnetic field. In order to allow comparison of traces obtained with different gate voltages, magnetoresistance is converted numerically into magnetoconductance, whereby the variations become of comparable
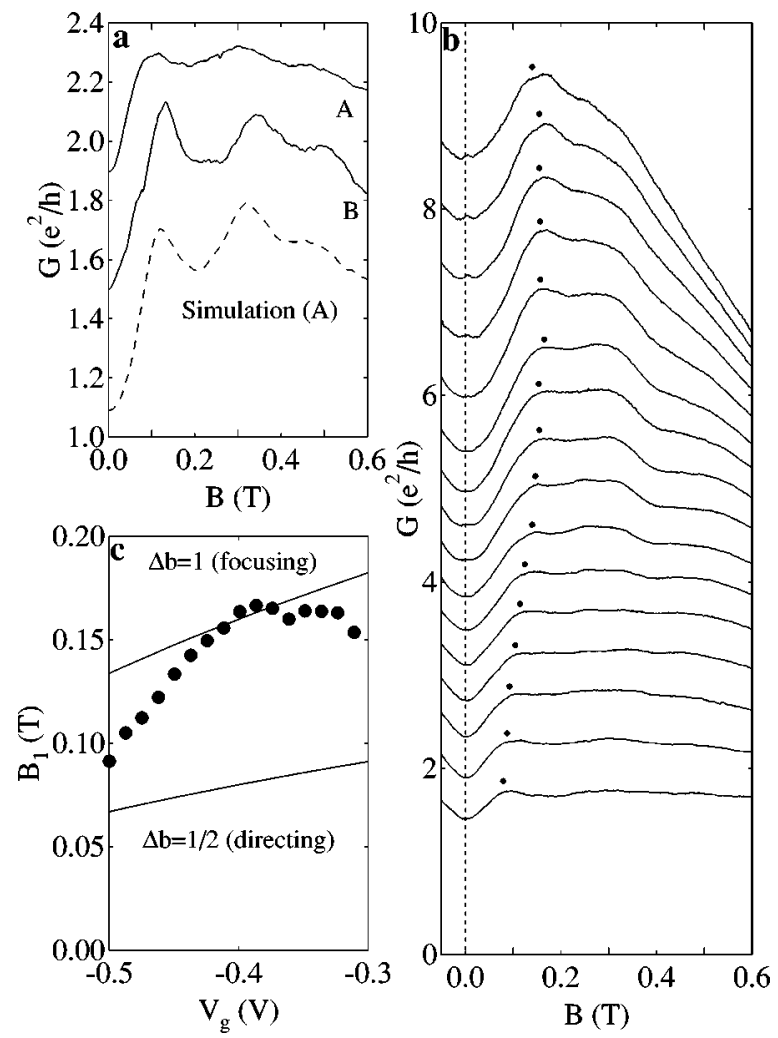

FIG. 2. (a) Magnetoconductance of samples A and B measured at $T=4.2 \mathrm{~K}$ (full lines) and a classical simulation (dashed line) using 100000 trajectories to compute each magnetoconductance point. The parameters of the simulation correspond to sample A. (b) The measured magnetoconductance of sample A shown for 16 gate voltages ranging from the formation voltage $V_{f}=-0.30 \mathrm{~V}$ (top curve) to the pinch-off voltage $V_{d}=-0.50 \mathrm{~V}$ (bottom curve). The position $B_{1}$ of the first conductance maximum (filled dots) is reduced from $0.18 \mathrm{~T}$ to $0.1 \mathrm{~T}$ as the gate voltage is made more negative. (c) $B_{1}$ is plotted against gate voltage. The full lines show the limiting periodicities calculated for $\Delta b=1$ (top line) and $\Delta b$ $=1 / 2$ (bottom line). $B_{1}$ is seen to move towards the $\Delta b=1 / 2$ limit as the gate voltage approaches pinch off.

magnitude in the studied range of gate voltages. Typical traces of conductance versus magnetic field are shown in Fig. 2(a). A focusing spectrum composed of a strong central $(B=0)$ conductance dip and one to two smaller peaks at higher magnetic fields is observed in all samples, in contrast to the continued sequence of peaks observed in perpendicular focusing geometries. ${ }^{6,7}$ In Fig. 2(b) the magnetoconductance traces for different gate voltages are shown for sample A. The position $B_{1}$ of the first maximum is seen to change from $0.18 \mathrm{~T}$ to $0.1 \mathrm{~T}$ as the gate voltage is changed from $-0.30 \mathrm{~V}$ to the pinch-off voltage $-0.50 \mathrm{~V}$. The two important observations are that the magnetic focusing spectrum is much weaker than found in perpendicular injection studies with comparable quality of 2D electron gases, ${ }^{6,7}$ and that the first peak position $B_{1}$ is reduced by nearly a factor of 2 as the gate voltage is approaching pinch off. Considering the long mean free path $(\approx 15 \mu \mathrm{m}$ in bulk) and the quality of the sample lithography [see Fig. 1(e)], it is clear that the quenching is not caused by imperfections or disorder in the electrostatic potential. We have compared the measured magnetoconductance curves to the result of a classical simulation of 
the electron transport in a disorder-free, soft-walled potential $^{9}$ with a triangular shape similar to the studied samples. An ensemble of trajectories is started outside one of the three openings with spatial and angular distributions according to the prescriptions suggested in Ref. 13. The transport coefficients can then be calculated by counting the number of trajectories leaving each of the three openings, whereby the conductance is obtained from a LandauerBüttiker formula. ${ }^{9,11,12}$ The result of such a calculation is compared to the measurements in Fig. 2. A strong damping of the simulated magnetic focusing spectrum similar to that of the experiment is evident, even though the simulation has been carried out with a disorder-free potential.

Below we will introduce a simple, quantitative model for the transmission through a generalized double slit in order to show that the quenching behavior occurs even in ideal, hardwalled double slits with negligible widths of the openings provided the injection angles are skewed, and that the variations of the first maximum position $B_{1}$ (or the focusing period) is in fact related to the detailed angular distribution of the injected trajectories.

\section{THEORY OF TRANSMISSION IN GENERALIZED DOUBLE-SLIT GEOMETRY}

Consider two openings through a specularly reflecting wall that are separated by a distance $L$. If the magnetic field is zero and the system is free of disorder, the electron beam injected from the emitter will propagate in the direction perpendicular to the wall, and will therefore not reach the other opening (collector), hence the current through the openings will be zero. If a magnetic field $B$ is then applied perpendicular to the plane, the beam can be deflected towards the collector, whereby the conductance increases for one choice of magnetic field direction. In a magnetic field an electron with initial angle $\alpha$ will perform skipping orbits with reflections at the points

$$
x=2 R_{c} \cos (\alpha) p, \quad p=1,2,3 \ldots,
$$

where $R_{c}$ is the cyclotron radius, and $p-1$ is the number of reflections needed to reach the point $x$. If a reflection point $x_{p}$ coincides with the collector, $x_{p}=L$, the electron is transmitted, leading to an enhancement of the conductance.

In the following we will consider the two limits of a narrow distribution and a broad distribution of angles, related to the already mentioned aiming and focusing mechanisms, respectively. By taking all electrons to inject in a narrow beam perpendicular to the reflecting wall, $\alpha=0$ for all trajectories, we trivially arrive at a periodicity given by Eq. (1). However, trajectories injected from a point contact generally have a broad angular distribution, ${ }^{2,3}$ which is symmetric around the injection axis. The distance $\Delta x=x_{p}-x_{p-1}$ between successive reflection points of a single electron trajectory will then depend on its injection angle $\alpha$ and the cyclotron radius $R_{c}$ as described by Eq. (2). A certain distribution $P(\alpha)$ of injection angles thus leads to a certain distribution in $\Delta x$. It is therefore not immediately obvious how a sharp periodic conductance can emerge from a broad distribution. This is related to a focusing effect of classical origin. As it turns out in the case of a broad distribution of injection angles, a magnetic field will lead to a high density of trajectories (caustics) at the same reflection points $2 R_{c} p(p=1,2,3, \ldots)$ as for the aiming effect. This is illustrated in Fig. 1(b) where classical electron trajectories clearly form dense spots near the wall at equidistant points, despite the uniform angular distribution of the emitted beam. Therefore, the distance between focal points is $2 R_{c}$ independent of the width of the angular distribution. Since the aiming and focusing mechanisms lead to the same transmission condition, the two mechanisms are practically indistinguishable.

This is, however, only true for the conventional case of a perpendicular injection axis. In Figs. 1(c) and 1(d) we have illustrated a narrow and broad angular distribution injected in a double slit where the injection axis is tilted by $\alpha_{0}=\pi / 3$ with respect to the normal of the wall. For the narrow distribution the reflection points are spaced by $R_{c} p$ as predicted by Eq. (2), while no clear focusing spots are observed for a broad distribution. Thus, for the skewed geometry the angular distribution is important, in contrast to the previous case. In order to investigate this further we derive an expression for the conductance of a double-slit with arbitrary collector width and injection angle direction and distribution.

Consider a double-slit geometry with specularly reflecting hard walls and openings of width $W$. It is convenient to introduce the dimensionless field $b=L / 2 R_{c}$, whereby the magnetoconductance becomes periodic in $b$ [see Eq. (2)]. We intend to calculate the probability $T_{d}$ of "direct" transmission from emitter to collector, by which we mean transmission via any number of specular reflections on the hard walls, i.e., ballistic transport. By convention the transmission probability is scaled by the number of transmitted channels $N$, with $T_{d}=N$ corresponding to full transmission. The conductance of two identical point contacts in series with a probability $T_{d}$ of direct transmission through the collector is given by ${ }^{4}$

$$
G=\frac{1}{2} G_{\mathrm{QPC}}\left(1+\frac{T_{d}}{N}\right),
$$

where $G_{\mathrm{QPC}}=2\left(e^{2} / h\right) N$ is the conductance of the individual point contact, and the factor of 2 is due to spin degeneracy. In the classical limit, ${ }^{13}$ the channel index $N$ can be approximated by $N \simeq k_{F} W / \pi$.

The transmission probability can be written as an integral over angle $\alpha$ :

$$
T_{d}(b)=\int_{-\pi / 2}^{\pi / 2} d \alpha P(\alpha) T_{d}(b, \alpha)
$$

where $T_{d}(b, \alpha)$ is the scaled probability of transmission of a trajectory with the angle $\alpha$, and $P(\alpha)$ is the initial angular distribution of the injected electron beam. An electron is transmitted if there exists a reflection point $x_{t}$ where $L$ $-W / 2<x_{t}<L+W / 2$. From Eq. (2) we can define an integer function $p(x)$ counting the number of reflection points between the emitter and some point $x$ along the wall,

$$
p(x)=\operatorname{Int}\left(\frac{x}{(L / b) \cos \alpha}+1\right) .
$$

It is seen from Eq. (2) and Eq. (5) that the first reflection point that has reached the nearest edge $L-W / 2$ of the open- 
ing is $x_{t}=(L / b) \cos (\alpha) p(L-W / 2)$, so that the angular transmission function can be written as

$$
\frac{T_{d}(b, \alpha)}{N}= \begin{cases}1 & \text { if } \quad x_{t}<L+W / 2 \\ 0 & \text { otherwise }\end{cases}
$$

The integral (4) can now be evaluated numerically by choosing an appropriate angular distribution $P(\alpha)$ and the relative point contact width $W / L$. The two-terminal conductance is obtained from Eq. (3). With the classical angular distribution $P(\alpha)=\frac{1}{2} \cos (\alpha)$ for a "hole in the wall" type opening, ${ }^{3}$ the results given in Ref. 6 for finite as well as infinitesimal openings $W / L$ are reproduced.

The cosine distribution is, however, unrealistic in many practical situations due to collimation of the electron beam by passage through a narrow contact. ${ }^{3}$ For an idealized point contact geometry in the adiabatic approximation ${ }^{3}$ the injection angular distribution is described as a cosine distribution $P(\alpha)=(f / 2) \cos (\alpha)$ truncated at the angles $\alpha_{\max }=$ $\pm \arcsin (1 / f)$, with $f$ depending on the shape and barrier height of the opening. ${ }^{3}$ Experiments and simulations, ${ }^{2}$ however, indicate a less abrupt cutoff at $\alpha_{\max }$ than implied by the adiabatic approximation. This can conveniently be taken into account by convoluting the truncated cosine with a Gaussian function of suitable width $w$. To model a skewed geometry, the symmetry axis of the angular distribution is offset by $\alpha_{0}$, which leads to the following angular distribution:

$$
P(\alpha)=\frac{(f / 2) \cos \left(\alpha-\alpha_{0}\right)}{1+\exp \left[4 w^{-1}\left(\left|\alpha-\alpha_{0}\right|-\alpha_{\max }\right]\right.},
$$

which for truncation angle $\alpha_{\max }>w$ gives a cosine distribution with soft truncations at $\alpha_{0} \pm \alpha_{\max }$. Using $w \approx 0.3$ and $\alpha_{0}=0$ distributions similar to the experimental results of Ref. 2 are reproduced.

\section{RESULTS}

We have compared $T_{d}(B)$ for perpendicular [Fig. 3(a)] and skewed [Fig. 3(b)] injection angles as obtained from the described model. In each case, the transmission has been calculated for nine angular distributions with different cutoff angles $a_{\max }$ ranging from 0 to $\pi / 3$ in steps of 0.1 radian. The distributions were then softened as described above, using $w=0.3$. The bottom curves of Figs. 3(a) and 3(b) are for the smallest cutoff angles $\alpha_{\max }$ corresponding to the pinch-off limit, while the top curves correspond to large $\alpha_{\max }$ and wide openings. The focusing spectra can be directly compared to those in Fig. 2 for sample $A$ due to the simple relation (3) of $G$ and $T_{d}$.

The perpendicular injection angle geometry exhibits clear focusing spectra with the period $\Delta b=1$ independent of the width of the angular distribution. For the skewed geometry an altogether different behavior is observed: with a broad distribution (top curves), the focusing spectra of the skewed and perpendicular angle geometries are similar, as a flat distribution per definition does not have any particular direction. As the distribution is narrowed down, the focusing spectrum is gradually quenched. For a very narrow distribution (bottom curve) a clear focusing spectrum is again recovered, however, with a smaller period. A closer study reveals
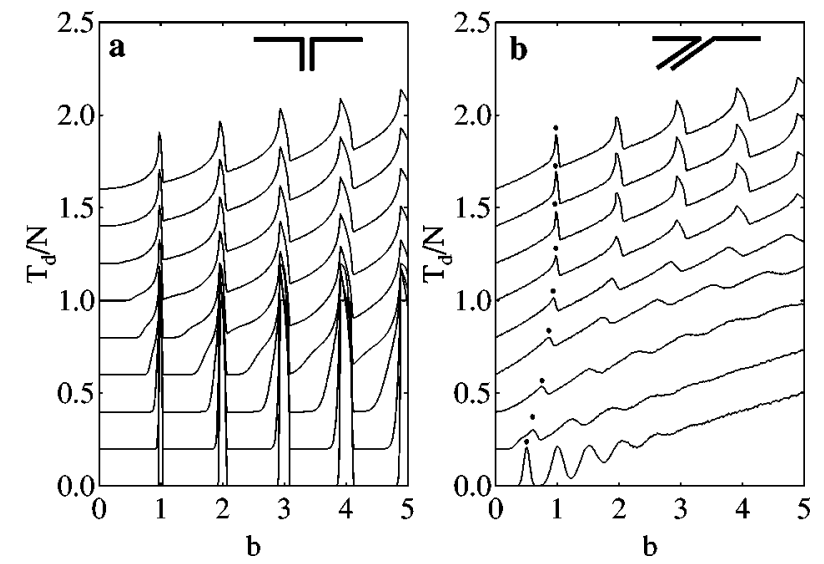

FIG. 3. (a) The direct transmission probability $T_{d} / N$ for a perpendicular geometry, plotted against the dimensionless field $b$. The spectra are plotted for nine different widths of the angular distribution, with the top curve representing a broad distribution and the bottom curve a narrow distribution. The spectra are remarkably similar, despite the radical change of the injection angle distribution function. (b) For a skewed injection angle geometry, a strong quenching occurs as the distribution is narrowed. As the distribution becomes very narrow, the focusing spectrum reappears with the half period $\Delta b=1 / 2$.

that the field period is changing smoothly from $\Delta b=1$ to $\Delta b=1 / 2$ as the injection angle distribution is narrowed, corresponding to the field period given by Eq. (2) with $\alpha_{0}$ $=\pi / 3$. This behavior can be understood as a gradual transition from the focusing behavior characteristic of a broad beam to the aiming behavior of a narrow beam. As a consequence the amplitudes of the focusing spectra in the intermediate regime are significantly reduced.

We have plotted the experimentally found peak positions $B_{1}$ as a function of gate voltage in Fig. 2(c). For the $\alpha_{0}$ $=\pi / 3$ geometry, the peak positions $B_{1}=(2 \hbar \sqrt{2 \pi n} / e L) \Delta b$ are limited by the cases of pure aiming $(\Delta b=1 / 2)$ and pure focusing $(\Delta b=1)$. Due to the decreasing carrier density with decreasing gate voltage, these limiting curves depend on gate voltage. Sample A has previously been estimated to have carrier densities changing linearly from $1.3 \times 10^{15} \mathrm{~m}^{-2}$ at the formation to $0.7 \times 10^{15} \mathrm{~m}^{-2}$ at pinch off. ${ }^{9}$ The limiting curves for $\Delta b=1$ and $\Delta b=1 / 2$ are plotted as full lines in Fig. 2(c). The experimental points are seen to shift away from the $\Delta b=1$ curve towards the $\Delta b=1 / 2$ curve as the gate voltage is changed from the formation voltage [wide $P(\alpha)$ ] to the pinch-off voltage [narrow $P(\alpha)$ ], similar to the calculation shown in Fig. 3(b). A more accurate calculation of the periodicity is difficult, since the exact shape of the angular distribution as a function of gate voltage is not known.

Finally, we consider how the detailed shape of the angular distribution affects the focusing spectra. In the inset of Fig. 4(a) we have plotted the truncated cosine distribution corresponding to an ideal adiabatic model ${ }^{3}$ or a Gaussian softened distribution similar to those found by simulations and experiments. ${ }^{2}$ The distribution width is in both these cases chosen to match the distributions obtained in Ref. 2. The focusing spectra obtained with these distributions and the relative opening width $W / L=0.1$ are shown in Fig. 4(a) and Fig. 4(b). With a perpendicular injection angle geometry the focusing spectra for the two distributions are nearly identical. 

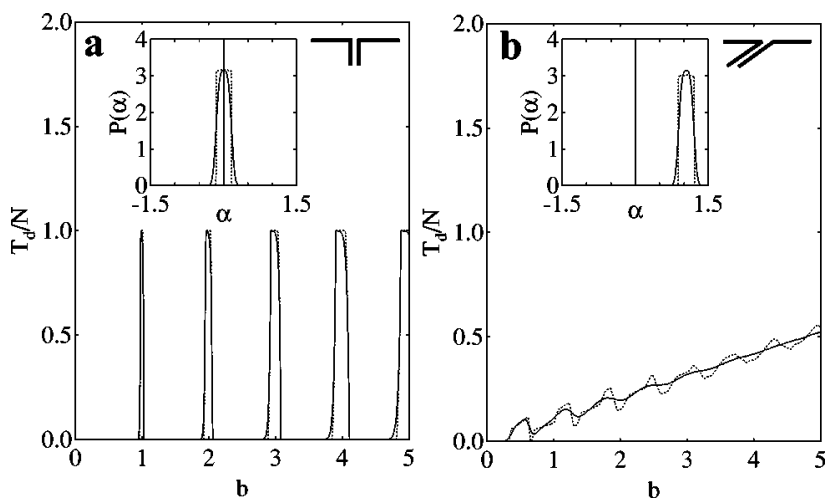

FIG. 4. (a) The main figure shows two spectra calculated for a perpendicular geometry, with angular distributions of the same width, but different shape. The dotted curve is an abrupt truncation as in the adiabatic model (see text) while the full line is the same distribution, only softened by a Gaussian of width $w=0.3$. The shape of the injection angle distribution is seen not to influence the focusing spectrum significantly. (b) For a skewed geometry with $\alpha_{0}=\pi / 3$, the amplitude is dramatically reduced for a softened distribution compared to the abruptly truncated distribution.

In contrast, the spectra for a skewed injection angle geometry are rather different. The periodicity is developed in the case of the truncated cosine distribution while nearly quenched for the softened distribution, despite the fact that the distributions have the same width. The shape of the distribution thus affects the amplitude and shape, but not the period of the focusing spectra, in the case of a skewed injection angle geometry.

\section{SUMMARY AND DISCUSSION}

We have fabricated and studied experimentally two samples in the shape of a triangle with open corners, which, for the purpose of the new effect described here, represents a double-slit geometry with skewed injection angle $\alpha_{0}=\pi / 3$. We observe a strong quenching of the magnetic focusing spectrum compared to conventional perpendicular geometries, and a reduction by nearly a factor of 2 of the field where the first conductance maximum occurs, as the injection opening is narrowed towards pinch off. Using a theoretical model we explain in terms of the interplay of two competing transport mechanisms. The focusing mechanism dominates the spectrum of the open billiard giving a periodicity $\Delta b=1$, while the aiming mechanism dominates the nearly closed billiard with the periodicity $\Delta b=\cos \left(\alpha_{0}\right)$. Therefore, a quenching of the spectrum as well as a transition of the first conductance maximum from 1 to $\cos \left(\alpha_{0}\right)$ in dimensionless field units $b=L / 2 R_{c}$. Finally, we note from the model calculations that the shape of the angular distribution is extremely important for the amplitude of the focusing spectra. In conclusion, strong quenching of the focusing effect occurs under the conditions of skewed aiming angles and realistic, softened angular distributions.

We have considered possible implications of these observations. Classical magnetoballistic effects play an important role in the interpretation of quantum interference effects in quantum billiards. ${ }^{9,10}$ Based on our results, we find that samples can be designed with deliberately weak classical fluctuations, thus allowing the quantum contributions to be observed more clearly. Another related aspect is the wellknown appearance of sidelobes in the angular distributions ${ }^{14}$ due to angular momentum quantization in the narrow emitter opening. In fact, each sidelobe acts as a separate skewed injection cone contributing to the magnetoconductance with its own periodicity given by Eq. (2), which we, from simulations, find leads to rather complicated classical contributions to the conductance. Though this is a classical effect, its dependence on momentum quantization will result in a larger temperature dependence than usually observed at the lowest temperatures. Finally, we consider magnetic focusing of composite fermions, ${ }^{8}$ which were originally introduced to account for electron-electron interactions in a $2 \mathrm{DEG}$ at high magnetic fields. The effective field experienced by these quasiparticles depends on the electron carrier density, and is therefore sensitive to local carrier density fluctuations, ${ }^{15}$ such as in the narrow emitter opening. The resulting field variations will effectively skew the average injection axis, which could lead to conductance asymmetries. ${ }^{15}$

\section{ACKNOWLEDGMENTS}

We are thankful to M. Persson at Chalmers University of Technology, who performed the $e$-beam exposure, to C. B. Sørensen at the $\emptyset$ rsted Laboratory for providing us with high quality sample material, and to R. Jensen for technical assistance. The work was supported by III-V NANOLAB in a MIC/NBIfAFG collaboration, by CNAST, by SNF, and by Novo Nordisk foundation.
${ }^{1}$ M. L. Roukes, A. Scherer, S. J. Allen, Jr., H. G. Craighead, R. M. Ruthen, E. D. Beebe, and J. P. Harbison, Phys. Rev. Lett. 59, 3011 (1987); C. J. B. Ford, T. J. Thornton, R. Newbury, M. Pepper, H. Ahmed, D. C. Peacock, D. A. Ritchie, J. E. F. Frost, and G. A. C. Jones, Phys. Rev. B 38, 8518 (1988).

${ }^{2}$ L. W. Molenkamp, A. A. M. Staring, C. W. J. Beenakker, R. Eppenga, C. E. Timmering, J. G. Williamson, C. J. P. M. Harmans, and C. T. Foxon, Phys. Rev. B 41, 1274 (1990).

${ }^{3}$ C. W. J. Beenakker and H. van Houten, Phys. Rev. B 39, 10445 (1989).

${ }^{4}$ D. A. Wharam, M. Pepper, H. Ahmed, J. E. F. Frost, D. G. Hasko, D. C. Peacock, D. A. Ritchie, and G. A. C. Jones, J. Phys. C 21, L887 (1988); P. E. Beton, B. R. Snell, P. C. Main,
A. Neves, J. R. Owers-Bradley, L. Eaves, M. Henini, O. H. Hughes, S. P. Beaumont, and C. D. W. Wilkinson, J. Phys.: Condens. Matter 1, 7505 (1989).

${ }^{5}$ J. Spector, J. S. Weiner, H. L. Stormer, K. W. Baldwin, L. N. Pfeiffer, and K. W. West, Surf. Sci. 263, 240 (1992).

${ }^{6}$ H. van Houten, C. W. J. Beenakker, J. G. Williamson, M. E. I. Broekaart, P. H. M. van Loosdrecht, B. J. van Wees, J. E. Mooji, C. T. Foxon, and J. J. Harris, Phys. Rev. B 39, 8556 (1989).

${ }^{7}$ J. Spector, H. L. Stormer, K. W. Baldwin, L. N. Pfeiffer, and K. W. West, Surf. Sci. 228, 283 (1990).

${ }^{8}$ A recent overview of composite fermion experiments: R. L. Willet, Adv. Phys. 46, 447 (1997).

${ }^{9}$ P. Bøggild, A. Kristensen, H. Bruus, S. M. Reimann, and P. E. 
Lindelof, Phys. Rev. B 57, 15408 (1998).

${ }^{10}$ C. M. Marcus, A. J. Rimberg, R. M. Westervelt, P. F. Hopkins, and A. C. Gossard, Phys. Rev. Lett. 69, 506 (1992); L. Christensson, H. Linke, P. Omling, P. E. Lindelof, I. V. Zozoulenko, and K.-F. Berggren, Phys. Rev. B 57, 12306 (1998); J. P. Bird, D. K. Ferry, R. Akis, Y. Ochiai, K. Ishibashi, Y. Aoyagi, and T. Sugano, Europhys. Lett. 35, 529 (1996); I. V. Zozoulenko, R. Schuster, K.-F. Berggren, and K. Ensslin, Phys. Rev. B 55, R10 209 (1997); M. Persson, J. Petterson, B. von Sydow, P. E. Lindelof, A. Kristensen, and K. F. Berggren, ibid. 52, 8921 (1995).
${ }^{11}$ M. Büttiker, Phys. Rev. B 33, 3020 (1986).

${ }^{12}$ P. Bøggild, A. Kristensen, P. E. Lindelof, S. M. Reimann, C. B. Sørensen, and M. Persson (unpublished).

${ }^{13}$ H. U. Baranger, D. P. V. DiVincenzo, R. A. Jalabert, and A. D. Stone, Phys. Rev. B 44, 10637 (1991).

${ }^{14}$ K. L. Shepard, M. L. Roukes, and B. P. van der Gaag, Phys. Rev. B 46, 9648 (1992).

${ }^{15}$ J. H. Smet, D. Weiss, R. H. Blick, G. Lütjering, K. von Klitzing, R. Fleischmann, R. Ketzmerick, T. Geisel, and G. Weimann, Phys. Rev. Lett. 77, 2272 (1996). 